ÉXITO Y SATISFACCIÓN LABORAL EN MUJERES DUEÑAS DE PYMES EN LA CIUDAD DE MÉRIDA, YUCATÁN.

\title{
Éxito y Satisfacción Laboral en Mujeres Dueñas de Pymes en la Ciudad de Mérida, Yucatán
}

\section{Success and Satisfaction in Working Women who Own SMEs in Merida, Yucatan}

Flores Novelo, Anel* ${ }^{*}$ Arroyo Solis, Shaira Andrea**, Camacho Gómez, Manuela del Socorro***

\begin{abstract}
*Doctora en Ciencias de la Administración, por la Universidad Nacional Autónoma de México Profesor de tiempo completo la Facultad de Contaduría y Administración de la Universidad Autónoma de Yucatán. ORCID: https://orcid.org/0000-0003-2567-8769.

**Bachiller, pasante de la Licenciatura en Administración de la Universidad Autónoma de Yucatán. Email: andreaarroyosolis@gmail.com, ORCID: https://orcid.org/0000-0003-4914-0402.

${ }^{* * *}$ Doctora en Educación Internacional. Profesora Investigadora de la División Académica de Ciencias Económico Administrativas. Universidad Juárez Autónoma de Tabasco. Email: manuela.camacho@ujat.mx, ORCID: https://orcid.org/0000-0003-3632-064X.
\end{abstract}

Correo para recibir correspondencia: anel.flores@correo.uady.mx

Fecha de recibido: 19 de noviembre de 2020

Fecha de aceptación: 11 de enero de 2021

FLORES-NOVELO A., ARROYO-SOLIS S. A. CAMACHO-GÓMEZ M. DEL S. 
ÉXITO Y SATISFACCIÓN LABORAL EN MUJERES DUEÑAS DE PYMES EN LA CIUDAD DE MÉRIDA, YUCATÁN.

RESUMEN

OBJETIVO: Evaluar el nivel de éxito y satisfacción laboral que cuentan las mujeres dueñas de Pequeñas y Medianas Empresas (PYMES) en Mérida, Yucatán, así como identificar los factores que afectan los niveles de satisfacción, como afectan a las dueñas de las pymes y mostrar los diferentes tipos de satisfacciones que pudieran tener cada una de las dueñas en contraste con sus diferentes estilos de vida.

MATERIAL Y MÉTODO: Los resultados presentados en este trabajo fueron producto de una investigación cuantitativa con enfoque deductivo. La población de estudio fueron las 85 empresas pertenecientes a la Asociación Mexicana de Mujeres en Yucatán, la cual es una asociación civil de mujeres empresarias ubicada en el estado de Yucatán. El instrumento fue desarrollado a partir del cuestionario del estudio CISMS II (Collaborative International Study Of Managerial Stress) de Poelmans (2003) y el Centro de Investigación de IAE-Business School. EI CISMS II es una herramienta estadísticamente validada.

RESULTADOS: Los resultados muestran que las mujeres que tienen un hijo y se encuentran en el rango de 50 años o más son las que poseen un nivel de satisfacción laboral más alto. Por otro lado, los resultados apuntan a que las mujeres tienden a tener una mejor satisfacción personal cuando se convierten en madres y el trabajo doméstico no afecta directamente a su trabajo.

CONCLUSIONES: Con base a la información obtenida, se puede observar que en la ciudad de Mérida Yucatán las mujeres cuentan con un alto nivel de satisfacción con relación "EmpresaPersona" y que, al contrario de otros estudios, en la ciudad de Mérida las mujeres que sienten más satisfacción son aquellas que ya se establecieron en su campo y las cuales cuentan con familia.

PALABRAS CLAVE: Satisfacción laboral. Pymes. Mujeres. Empresarial.

\section{ABSTRACT}

OBJECTIVE: To evaluate the level of success and job satisfaction that women owners of Small and Medium Enterprises (SMEs) have in Merida, Yucatan, as well as to identify the factors that affect satisfaction levels, the way they affect the owners of SMEs and to show the different types of satisfaction that each of the owners could have in contrast to their different lifestyles. 
ÉXITO Y SATISFACCIÓN LABORAL EN MUJERES DUEÑAS DE PYMES EN LA CIUDAD DE MÉRIDA, YUCATÁN.

MATERIAL AND METHOD: The results of this is a quantitative deductive research showed that 85 enterprises were part of the Mexican Association of Women in Yucatan, which is an association for entrepreneur women located in the state of Yucatan. The instrument was developed from the CISMS II questionnaire [Collaborative International Study Of Managerial Stres] by Poelmans (2003) and the Research Center (from IAE-Business School). The CISMS II is a validated statistics tool.

RESULTS: The results show that women who have a child and are in the range of 50 years or more have a higher level of job satisfaction. On the other hand, the results suggest that women tend to have better personal satisfaction when they become mothers and housework does not directly affect their work.

CONCLUSIONS: Based on the information obtained, it can be observed that in the city of Merida, Yucatán women have a high level of satisfaction with the "Company-Person" relationship and, contrary to other studies, women who feel more satisfaction are those who have already established themselves in their field and who have families.

KEY WORDS: Laboral satisfaction. SMEs. Women. Entrepreneurial.

\section{INTRODUCCIÓN}

La búsqueda de una mayor igualdad en el ámbito de la economía surge del auge de feminismo que está ganando espacios notable, tanto en países occidentales como en el resto del mundo, por lo cual la tendencia de las empresas es procurar la equidad para ser competitivos en materia de Responsabilidad Social Empresarial (RSE). También ha tenido repercusiones en la esfera de la política y la legislación, por ejemplo, en la ley orgánica española existen una serie de acciones que fomentan la presencia equilibrada de ambos géneros en los consejos de administración, dichas acciones suelen estar incluidas en el ámbito de responsabilidad social de las empresas (Vidal, 2010).

En la década del setenta, la incorporación de las mujeres al trabajo se fue consolidando a nivel global; sin embargo, en México esta tedencia se pudo observar más claramente hasta la década del noventa. Hacia la segunda década del siglo XXI el número de mujeres que concilian 
ÉXITO Y SATISFACCIÓN LABORAL EN MUJERES DUEÑAS DE PYMES EN LA CIUDAD DE MÉRIDA, YUCATÁN.

su desarrollo profesional con su rol cultural reproductivo y con respecto a la maternidad. Estos cambios se reflejan en los patrones de comportamiento de la Población Económicamente Activa (PEA) de las mujeres. Al respecto, algunas estimaciones muestran que la participación laboral registrada en la actividad económica de las mujeres pasó del orden de $16.4 \%$ y $19.6 \%$ durante 1970 y 1990 respectivamente, a 42.9\% durante el primer trimestre de 2016. Estos cambios son producto de la transición demográfica, proceso que entraña, en primer lugar, un descenso de las tasas de mortalidad y, posteriormente, una reducción de la fecundidad, lo que da lugar a cambios en la estructura de la población por edad y sexo, y ha sido un elemento decisivo del crecimiento de la fuerza laboral, y en especial para el proceso de feminización del trabajo (Ortíz et al., 2015).

De acuerdo con Rendón (2013), durante 1990, se aceleró el antes paulatino proceso de feminización de la fuerza de trabajo en México, lo que acentuó el incremento de la participación laboral femenina el cual se ha visto reforzado por la restructuración del aparato productivo y el deterioro de las condiciones económicas que privan en nuestro país. La incorporación masiva de la mujer en edad reproductiva al trabajo fue posible debido al descenso de la fecundidad, la cual se redujo de poco más de siete hijos en promedio durante la década de los setenta a 2.2 hijos, en primer trimestre de 2018, que, aunado a la postergación de la edad al matrimonio, redujo el número de años que las madres dedican a la crianza de sus hijos. De esta manera, el descenso de la fecundidad y la postergación de la edad para contraer matrimonio, han propiciado que las mujeres mexicanas de hoy puedan dedicar más tiempo al desarrollo de planes educativos o laborales que las mujeres de generaciones pasadas.

De acuerdo con datos del Instituto Nacional de Estadística y Geografía (INEGI, 2018), cada vez hay un número mayor de mujeres que se convierten en jefas de familia y proveedoras del sustento de sus hijos, ello debido a la propensión del aumento de las mujeres con hijos sin pareja (28.3\% al primer trimestre de 2018 ), mujeres solteras (con $9.2 \%$ ) y rupturas conyugales divorciadas $(2.5 \%)$, separadas $(6.4 \%)$ y viudas $(10.2 \%)$.

De estas estadísticas es importante destacar que la concepción de "jefe de familia" proviene de un discurso heteropatriarcal que considera a la pareja masculina como autoridad máxima de la familia y cuyo carácter no necesita explicación, ya que se suscribe en un supuesto ordenamiento natural y biológico. Por ello, para contabilizar a las jefas de familia sólo se consideran a las mujeres solas, pero con un criterio más amplio se puede estimar que en número de mujeres que sostienen hogares es mayor. 
ÉXITO Y SATISFACCIÓN LABORAL EN MUJERES DUEÑAS DE PYMES EN LA CIUDAD DE MÉRIDA, YUCATÁN.

En la Figura 1, se muestra la tasa de participación femenina al mercado laboral ne México, donde se observa que la incorporación de las mujeres al mercado laboral ha ido en aumento, siendo el cambio más notable, en las 5.6 millones de mujeres que participaron en la actividad económica durante el año 1990 en la producción de bienes y servicios, y que a partir de ahí empezaron a aumentar tales tasas de participación laboral de las mujeres hasta llegar a 20.1 millones durante el primer trimestre de 2018; lo que significa que mientras en 1990 de cada 100 mujeres en edad laboral, trabajaban alrededor de 16, en la actualidad representa más de la mitad, es decir, se desempeñan en el mercado laboral alrededor de 43 mujeres que son económicamente activas. Más aun, es fundamental reconocer que al primer trimestre de 2018 el creciente aumento de la participación femenina en México se explica en parte porque el trabajo en general está sujeto a los vaivenes de la actividad económica, caracterizada primordialmente por un menor dinamismo del Producto Interno Bruto (PIB) en México, lo que permite que la creciente incorporación de las mujeres, también se explique por el hecho de que la incertidumbre económica obliga necesariamente a la población y en especial a las mujeres a complementar el presupuesto familiar; en este sentido, cabe mencionar que el número de hogares mexicanos cuya jefatura es femenina asciende a 9 millones 266 mil 211, lo que significa que más de 29 de cada 100 hogares en el país lo encabeza una mujer (INEGI, 2018).

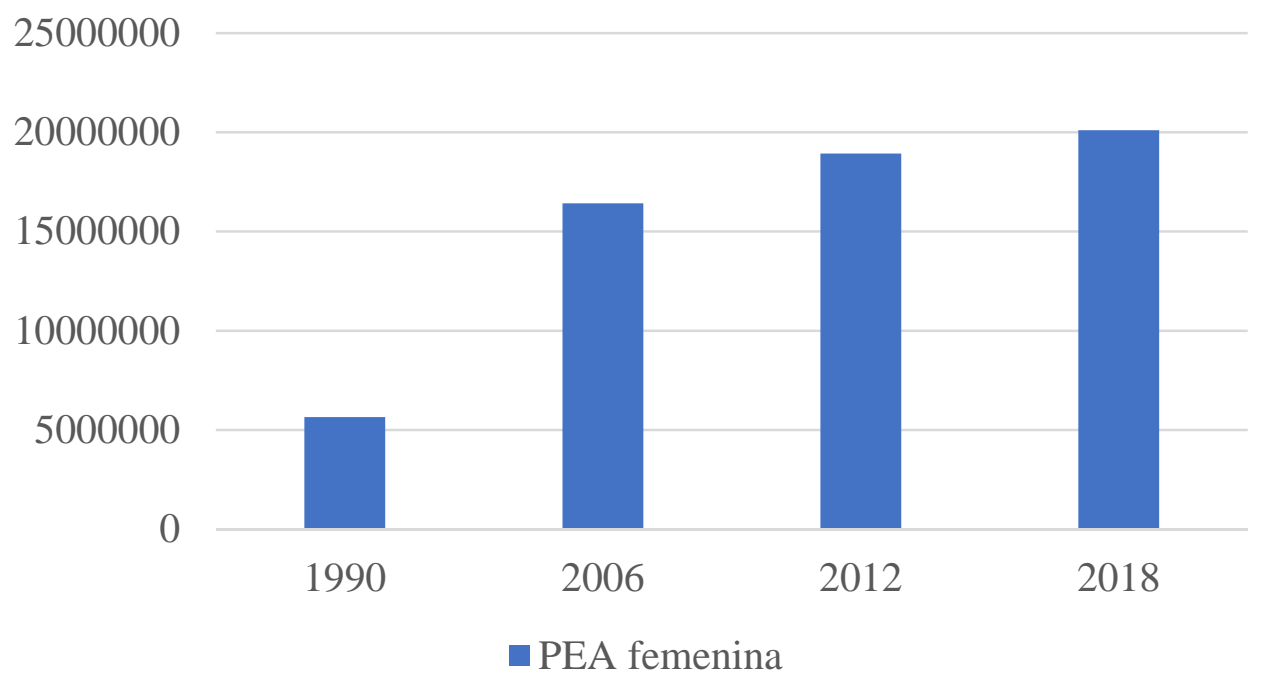

Figura 1. Tasa de participación femenina en México.

Fuente: Elaboración propia con datos del INEGI (2018).

Nota: El año 1990 se coloca con el fin de hacer referencia al momento en el cual se acentuó la participación de las mujeres en el mercado laboral de México, hasta las últimas fechas. 
ÉXITO Y SATISFACCIÓN LABORAL EN MUJERES DUEÑAS DE PYMES EN LA CIUDAD DE MÉRIDA, YUCATÁN.

Ante el panorama anterior, se planteó la pregunta de investigación: ¿Qué nivel de satisfacción personal y laboral cuentan las mujeres que son dueñas de Pequeñas y Medianas Empresas en Mérida, Yucatán? y como objetivo general: Evaluar el nivel de satisfacción laboral y personal que cuentan las mujeres dueñas de pymes en Mérida, Yucatán.

\section{MATERIAL Y MÉTODO}

Con base en los objetivos planteados, se realizó una investigación de tipo aplicada. De acuerdo con Márquez (2004), este tipo de investigaciones utilizan las teorías y los resultados de investigación realizados con anticipación para intentar entender la realidad estudiada, la cual en el presente estudio se circunscribe a la necesidad de conocer si el éxito y satisfacción laboral y personas en las mujeres dueñas de pymes en Mérida Yucatán es alto o no a través de un instrumento denominado: Encuesta de Satisfacción Laboral y Personal para Mujeres Dueñas de Pymes (ESLPMDP).

Con base a los procedimientos de acopio de información, el estudio que aquí se describe se clasifica como de campo, ya que la recolección de los datos primarios y secundarios se realizaron en la ciudad de Mérida, capital del estado de Yucatán. La recolección de la información se realizó por medio de una encuesta la cual fue administrada a las participantes con características juzgadas relevantes (ser mujer y ser dueña de una pyme en la ciudad de Mérida o sus alrededores) para los fines del presente trabajo.

Por último, con relación al momento y a la forma en que el fenómeno de interés fue abordado, la presente investigación es de naturaleza cuantitativa. La investigación cuantitativa implica el uso de herramientas informáticas, estadísticas, y matemáticas para obtener resultados. Es concluyente en su propósito, ya que trata de cuantificar el problema y entender qué tan generalizado está mediante la búsqueda de resultados proyectables a una población mayor (González, 1997).

La población de estudio fueron las 85 empresas pertenecientes a la Asociación Mexicana de Mujeres en Yucatán, la cual es una asociación civil de mujeres empresarias ubicada en el estado de Yucatán. Se logró un total de 48 observaciones para el presente trabajo, que considerando el tamaño de la población representa un 95\% de nivel de confianza y un 10\% de error muestral. 
ÉXITO Y SATISFACCIÓN LABORAL EN MUJERES DUEÑAS DE PYMES EN LA CIUDAD DE MÉRIDA, YUCATÁN.

Para la construcción del instrumento se tomó como referencia se tomó como base el cuestionario del estudio CISMS II (Collaborative International Study Of Managerial Stress), el cual fue resultado de la modificación del CISMS I realizada por Poelmans (2003), buscando enfocarse en el conflicto trabajo-familia en su doble dimensión (Poelmans, 2003) y luego por CONFYE (Centro de Investigación de IAE-Business School). EI CISMS II es una herramienta estadísticamente validada. Posteriormente se consultó el trabajo de Córdova (2012) para la confección de los dominios que conformaron el instrumento.

\section{RESULTADOS}

Se tomaron en cuenta diferentes factores para medir la satisfacción de cada una de las mujeres, uno de los principales factores fue el tamaño de la empresa de la que son dueñas. Como se puede observar en la Figura 2, de 48 dueñas entrevistadas 10 (20.83\%) cuentan con microempresas, teniendo de uno a cinco empleados, 17 (35\%) son pequeñas empresas, ya que tienen de 6 a 10 personas, 11 (22.91\%), fueron medianas empresas y, finalmente, un $20.83 \%$ entrarían en el rango de grandes empresas.

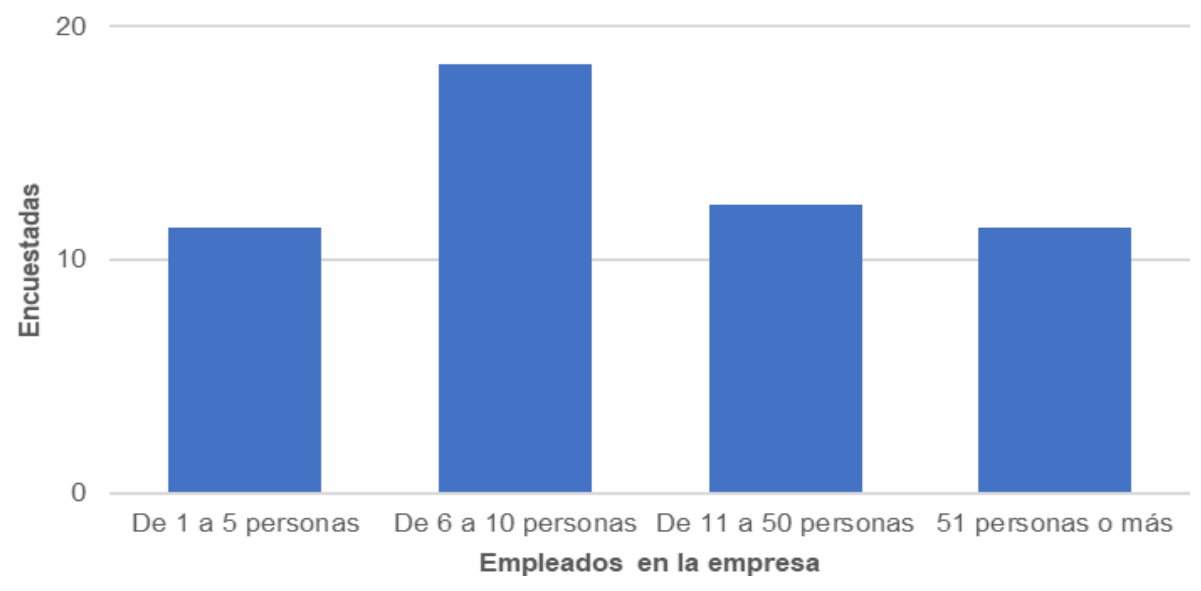

Figura 2. Tamaño de la empresa.

Fuente: Elaboración propia.

En cuanto a la edad de las empresarias sujetas de estudio, la mayor parte, un 48\%, tenía 50 años o más, seguidas de un $27 \%$ que tenían entre 41 y 50 , finalmente, un $25 \%$ tenían menos de 40 años (Figura 3). 
ÉXITO Y SATISFACCIÓN LABORAL EN MUJERES DUEÑAS DE PYMES EN LA CIUDAD DE MÉRIDA, YUCATÁN.

\section{Rango de edad}

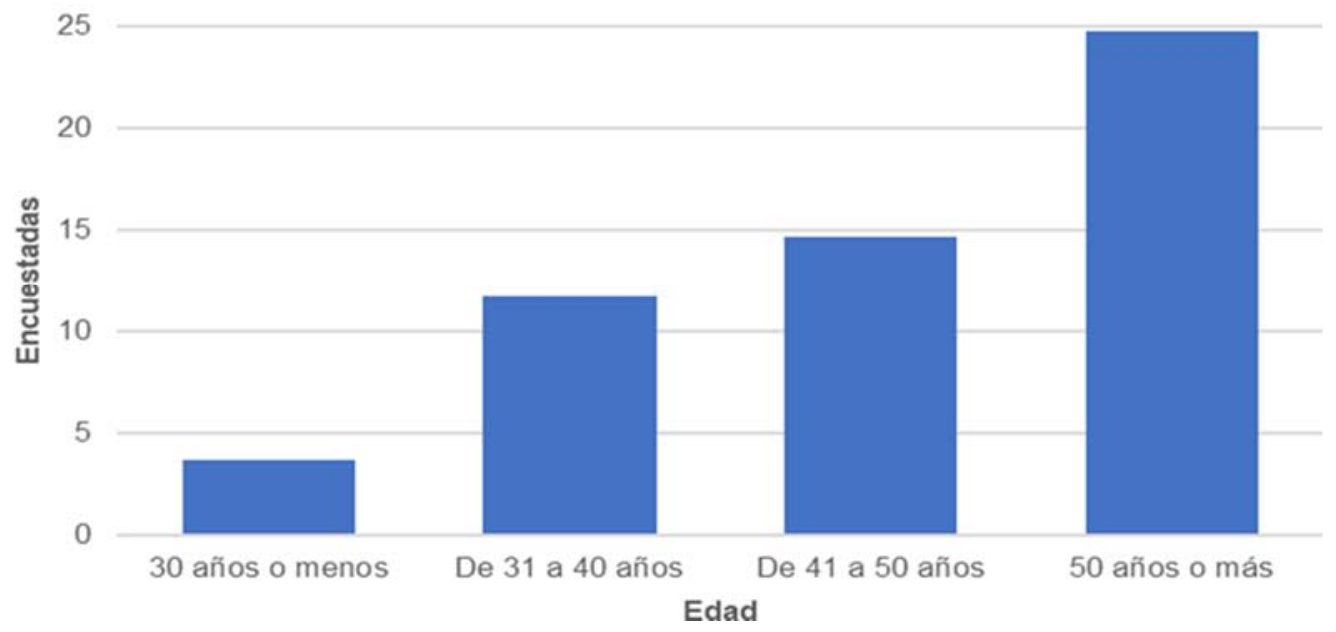

Figura 3. Edad de las dueñas.

Fuente: Elaboración propia.

Otro aspecto importante que se evaluó fue, si tenían hijos, y se encontró que un $65 \%$ tenían hijos. Asimismo, un $62.5 \%$ estaban casadas, en tanto que un $25 \%$ se encontraban solteras, un $4 \%$ de ellas manifestaron estar en unión libre y $8 \%$ cuentan con otro estado civil (viuda, divorciada, etc.).

En cuanto a la escolaridad, la mayor parte cuenta con licenciatura (50\%), seguida de $44 \%$ con posgrado y únicamente un $6 \%$ contaba con bachillerato o menos.

\section{Resultados en cuanto a nivel de satisfacción laboral y personal}

Para medir el nivel de satisfacción laboral y personal con el que contaban las dueñas de las pymes en Mérida Yucatán, se diseñó un instrumento con cuatro rubros y cada rubro contó con ocho reactivos.

Para un mayor entendimiento de esta etapa, se presentan los valores promedios obtenidos para cada uno de los rubros analizados en el instrumento, mismos que fueron medidos en una escala Likert de 1 al 5. La Tabla 1, contiene los descriptivos de los dominios del estudio y presenta características importantes de resaltar. Primero, se puede observar que el tema que obtuvo la puntuación más baja es el rubro 2 (Relación con el ambiente laboral de la empresa, $\dot{x}=2.1240$ ). 
ÉXITO Y SATISFACCIÓN LABORAL EN MUJERES DUEÑAS DE PYMES EN LA CIUDAD DE MÉRIDA, YUCATÁN.

Tabla 1

Estadística descriptiva de los niveles

\begin{tabular}{llcccc}
\hline Variables & & \multicolumn{4}{c}{ Estadística descriptiva } \\
\hline Temas & Nombre del tema & $\mathrm{N}$ & $\dot{\mathrm{x}}$ & $\mathrm{DE}$ \\
\hline Tema 1 & Relación empresa-persona & 48 & 4.2080 & 4.17326 & 4.154 \\
Tema 2 & Relación con el ambiente laboral de la empresa & 48 & 2.1240 & 2.00173 & 2.045 \\
Tema 3 & Satisfacción relación hogar-trabajo & 48 & 3.3941 & 3.15950 & 3.344 \\
Tema 4 & Satisfacción relación estilo de vida-trabajo & 48 & 3.6147 & 3.33205 & 3.774
\end{tabular}

Nota: Se puede observar, que el tema con mayor grado de satisfacción es el tema (Relación empresapersona $\dot{x}=4.2080$.

Fuente: Elaboración propia.

\section{Tipos de satisfacciones}

\section{Relación empresa-persona}

Con respecto a la relación empresa-persona, se obtuvieron 8 reactivos, los cuales son:

1. El trabajo que realizo dentro de la empresa es fundamental para su funcionalidad de esta.

2. Me siento satisfecha con las metas que se plantean en la empresa.

3. La cantidad de tareas que realizo en el trabajo me cansa y/o estresa.

4. Siento que el ritmo que manejo en el trabajo puede ser exagerado para el puesto que manejo.

5. He logrado la mayoría de los objetivos que me propuse al iniciar este trabajo.

6. Me he sentido emocionalmente estable al estar en este trabajo.

7. Las cosas que quiere conseguir en la vida las puede desarrollar teniendo este trabajo.

8. Me encuentro satisfecha con el desarrollo de mi carrera en la empresa.

Se puede observar en la Figura 4, de 48 mujeres encuestadas, 29 (60\%) mencionaron estar totalmente de acuerdo que el trabajo que realiza dentro de la empresa es fundamental para esta, 17 (35\%) mencionaron estar de acuerdo y 2 ni de acuerdo ni desacuerdo, en el rubro dos, $30(62.5 \%)$ mujeres manifestaron estar totalmente de acuerdo con el sentirse satisfecha con las metas que se plantean en la empresa, 17 (35\%) están de acuerdo y 1 (2\%) menciona ni estar de acuerdo ni en descuerdo, en rubro tres, 3 (6.25\%) mujeres mencionan estar totalmente de acuerdo al decir que el trabajo que realizan la cansan y/o estresan, 1 (2\%) mencionó estar de acuerdo, 3 (6.25\%) no están de acuerdo ni desacuerdo, 16 (33.3\%) manifiestan estar en 
ÉXITO Y SATISFACCIÓN LABORAL EN MUJERES DUEÑAS DE PYMES EN LA CIUDAD DE MÉRIDA, YUCATÁN.

desacuerdo y 25 (52\%) están totalmente en desacuerdo, en el rubro cuatro, 5 (10.41\%) mujeres mencionaron estar totalmente de acuerdo con que el ritmo que maneja en el trabajo puede ser exagerado para el puesto que maneja, 4 (8.33\%) dijeron que están de acuerdo, 24 (50\%) mencionaron ni estar de acuerdo ni desacuerdo, 9 (18.75\%) están en desacuerdo y $6(12.5 \%)$ manifestaron estar completamente desacuerdo, en el rubro cinco, 39 (81.25\%) mujeres mencionaron estar totalmente de acuerdo al decir que han logrado la mayoría de los objetivos que se propusieron al iniciar su trabajo, 6 (12.5\%) mencionaron estar de acuerdo, $2(4.16 \%) \mathrm{ni}$ de acuerdo ni desacuerdo y $1(2 \%)$ en desacuerdo, en el rubro seis, $34(70.83 \%)$ mujeres mencionan estar totalmente de acuerdo al sentirse emocionalmente estable al estar en su trabajo, 6 (12.5\%) están de acuerdo, 2 (4.16\%) mencionar ni estar de acuerdo ni en desacuerdo y $1(2 \%)$ mencionar estar en desacuerdo, en el rubro siete 37 (77\%) mujeres están totalmente de acuerdo con que las cosas que quieren conseguir en la vida las puede desarrollar teniendo ese trabajo, 8 (16.67\%) manifestaron estar de acuerdo y 2 (4.16\%) mencionaron ni estar de acuerdo ni en desacuerdo, en el último rubro, 40 (83\%) mujeres están totalmente de acuerdo en que se encuentran satisfechas con el desarrollo de su carrera en la empresa, 6 (12.5\%) mencionaron estar de acuerdo, 1 (2\%) mencionó ni estar de acuerdo ni desacuerdo y 1 (2\%) no está de acuerdo.

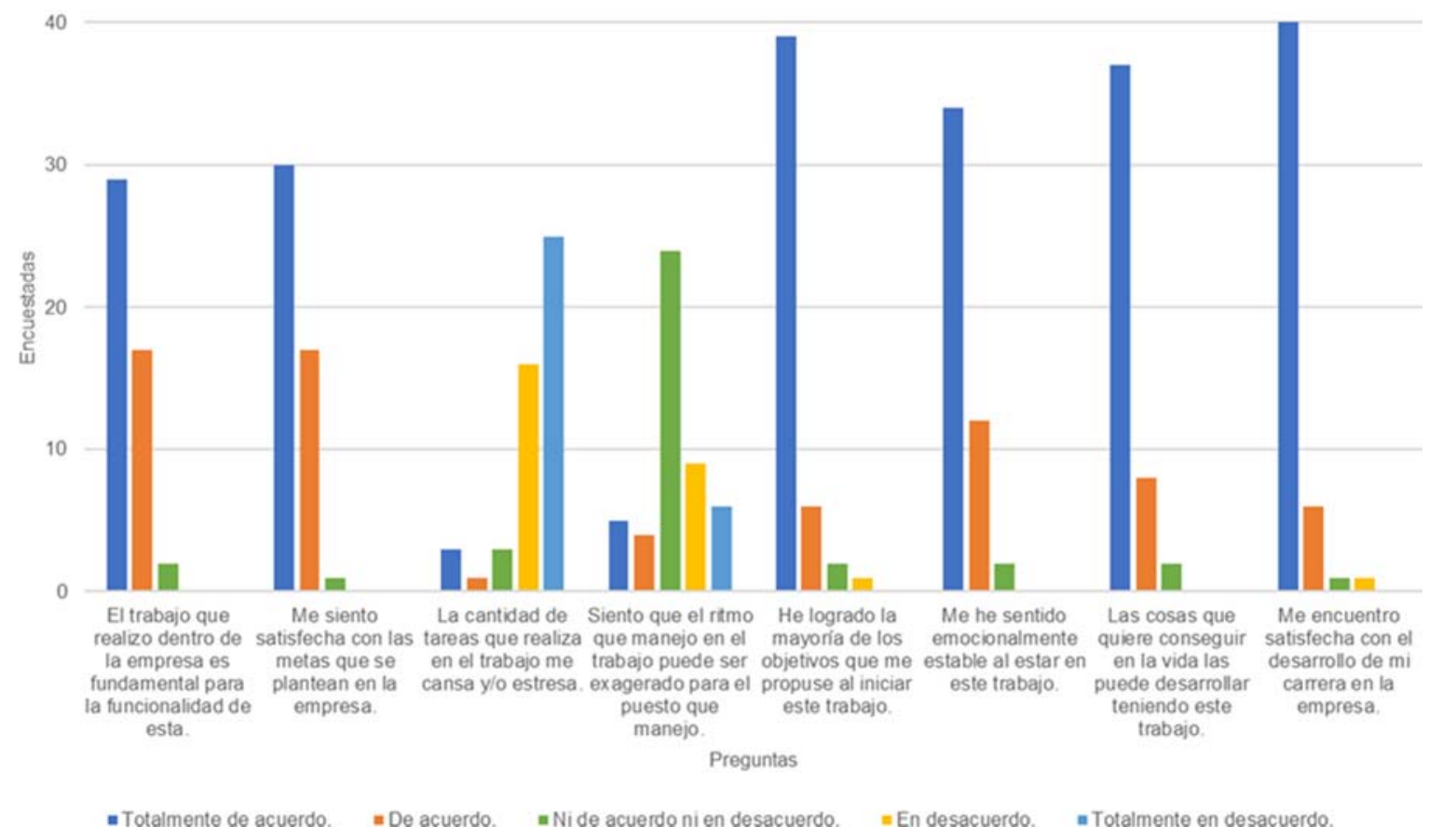

Figura 4. Relación empresa-persona.

Fuente: Elaboración propia.

FLORES-NOVELO A., ARROYO-SOLIS S. A. CAMACHO-GÓMEZ M. DEL S. 
ÉXITO Y SATISFACCIÓN LABORAL EN MUJERES DUEÑAS DE PYMES EN LA CIUDAD DE MÉRIDA, YUCATÁN.

\section{Relación con el ambiente laboral de la empresa}

Respecto a la relación con el ambiente laboral de la empresa se obtuvieron seis reactivos, los cuales fueron:

1. A mis compañeros de trabajo les interesa mi bienestar, fechas importantes y actividades de integración.

2. Los colaboradores de la empresa le dan importancia a los aportes y opiniones que hago a esta.

3. Me encuentro satisfecha con la forma en que se desempeñan los colaboradores de la empresa.

4. La relación con los compañeros y/o colaboradores de trabajo es buena.

5. Mi trabajo me ha permitido hacer nuevas amistades y/o un nuevo circulo social.

6. Los colaboradores escuchan y atienden mis requerimientos y necesidades.

Se puede observar en la Figura 5, de 48 mujeres encuestadas en el rubro uno, 2 (4.16\%) están totalmente de acuerdo en que sus compañeros de trabajo les interesa su bienestar, fechas importantes y actividades de integración, 8 (16.67\%) dicen estar de acuerdo, 23 (48\%) dicen ni estar de acuerdo ni desacuerdo, 10 (21\%) están en desacuerdo y 5 (10.41\%) totalmente en desacuerdo, en el rubro dos, se puede observar que 25 (52\%) mujeres están totalmente de acuerdo que los colaboradores de la empresa le dan importancia a los aportes y opiniones que hace de esta, 17 (35\%) están de acuerdo y 6 (12.5\%) ni están de acuerdo ni desacuerdo, en el rubro tres, 12 (25\%) mujeres manifestaron estar totalmente de acuerdo que se encuentra satisfecha con la forma en la que se desempeñan los colaboradores de la empresa, 4 (8.33\%) mencionaron estar de acuerdo, 25 (52\%) están ni de acuerdo ni desacuerdo y $6(12.5 \%)$ se encuentran en desacuerdo, en el rubro cuatro, 8 (16.67\%) mujeres están totalmente de acuerdo con que su relación con los compañeros de trabajo es buena, 20 (41.66\%) comentaron estar de acuerdo, 15 (31.25\%) mencionan ni estar de acuerdo ni desacuerdo y 5 (10.41\%) mencionaron estar en desacuerdo, en el rubro cinco, 16 (33.33\%) mujeres están totalmente de acuerdo de que su trabajo les permitió hacer nuevas amistades y/o circulo social, 20 (41.66\%) están de acuerdo con este rubro, 15 (31.25\%) comentaron que ni están de acuerdo ni en desacuerdo y 4 (8.33\%) comentaron que están en desacuerdo, en el último rubro, el número 6 (12.5\%) mujeres están totalmente de acuerdo con que los colaboradores escuchan y atienden sus requerimientos, 14 (29\%) de ellas están de acuerdo, 15 (31.25\%) ni están de acuerdo ni en desacuerdo, 8 están en desacuerdo y 5 (10.41\%) están totalmente en desacuerdo. 
ÉXITO Y SATISFACCIÓN LABORAL EN MUJERES DUEÑAS DE PYMES EN LA CIUDAD DE MÉRIDA, YUCATÁN.

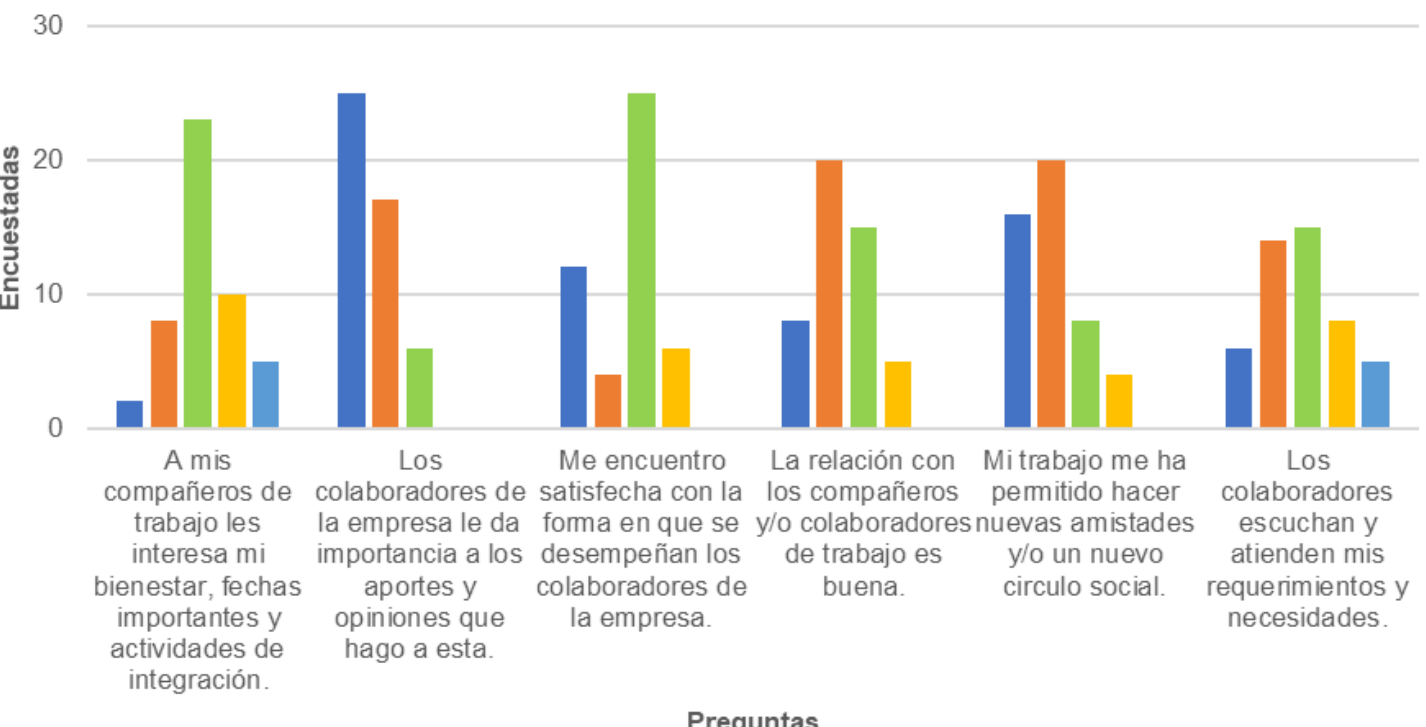

- Totalmente de acuerdo

ne acuerdo.

- Ni de acuerdo ni en desacuerdo

- En desacuerdo.

nTotalmente en desacuerdo.

Figura 5. Relación con el ambiente laboral de la empresa.

Fuente: Elaboración propia.

\section{Relación hogar-trabajo}

Con respecto a la relación hogar-trabajo, se obtuvieron 8 reactivos, los cuales son:

1. Los logros de mi trabajo me han permitido enriquecer mi relación de pareja.

2. Cumplir con mi trabajo es tan fácil como realizar tareas en el hogar.

3. Las horas de mi trabajo interfieren con el tiempo que debo dedicar a mis hijos/pareja/familia.

4. Cuando regreso a casa después del trabajo no tengo energía para realizar labores en la casa.

5. Los problemas familiares interfieren en mi trabajo.

6. Los problemas laborales interfieren en mi relación de familia.

7. Las preocupaciones del trabajo interfieren con mi relación de pareja.

8. En mi trabajo he aprendido cosas que me sirven para ser mejor madre/esposa/amiga.

Se puede observar en la Figura 6, en el rubro uno, que de 48 mujeres encuestadas 12 (25\%) mencionaron estar totalmente de acuerdo con el hecho de que los logros de su trabajo le han permitido enriquecer su relación de pareja, 15 (31.25\%) mencionan estar de acuerdo, 8 (16.66\%) ni de acuerdo ni desacuerdo y 3 (6.25\%) concuerdan en estar desacuerdo, en el rubro 
ÉXITO Y SATISFACCIÓN LABORAL EN MUJERES DUEÑAS DE PYMES EN LA CIUDAD DE MÉRIDA, YUCATÁN.

dos, 19 (39.58\%) mujeres mencionan estar totalmente de acuerdo que cumplir con su trabajo es tan fácil como realizar tareas en el hogar, 18 (37.5\%) están de acuerdo, 8 (16.66\%) ni de acuerdo ni desacuerdo y $3(6.25 \%)$ están en desacuerdo, para el rubro tres, 15 (31.25\%) mujeres están totalmente de acuerdo con que las horas de su trabajo interfieren con el tiempo que debe dedicar a mis hijos/pareja/familia, 21 (43.75\%) mencionan estar de acuerdo y 12 (25\%) ni están de acuerdo ni en desacuerdo, en el rubro cuatro, 12 (25\%) mujeres mencionan estar totalmente de acuerdo que cuando regresan a casa después del trabajo no tienen energía para realizar labores en la casa, 16 (33.3\%) mencionaron estar de acuerdo, 10 (20.83\%) ni de acuerdo ni desacuerdo, 6 (12.5\%) están en desacuerdo y 4 (8.33) en total desacuerdo, en el rubro cinco, 22 (45.83\%) mujeres mencionaron estar totalmente de acuerdo con que los problemas familiares interfieren en su trabajo, 16 (33.3\%) están de acuerdo y 10 (20.83\%) ni de acuerdo ni desacuerdo, en el rubro seis, 29 (60.42\%) mujeres mencionaron estar totalmente de acuerdo con que los problemas laborales interfieren en su relación de familia, 15 (31.25\%) mencionan estar de acuerdo y 4 (8.33\%) ni de acuerdo ni desacuerdo, en el rubro siete, 26 $(54.16 \%)$ mujeres mencionaron estar totalmente de acuerdo con que las preocupaciones del trabajo interfieren con su relación de pareja, 20 (41.66\%) están de acuerdo y 2 (4.16\%) ni de acuerdo ni desacuerdo, en el último rubro, 34 (70.83\%) mujeres mencionan estar totalmente de acuerdo al decir que en su trabajo ha aprendido cosas que le sirven para ser mejor madre/esposa/amiga, 12 (25\%) mencionan estar de acuerdo y 2 (4.16\%) ni de acuerdo ni desacuerdo.

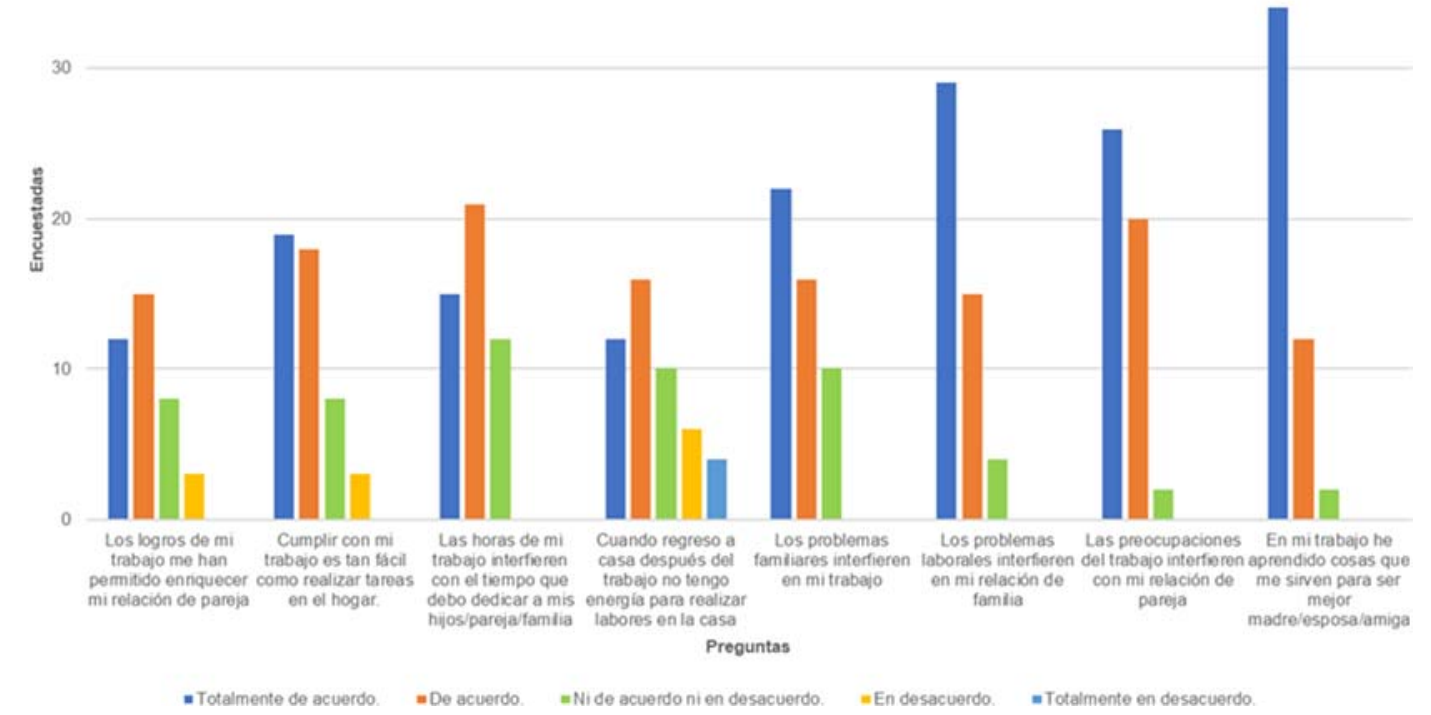

Figura 6. Relación hogar-trabajo.

Fuente: Elaboración propia. 
ÉXITO Y SATISFACCIÓN LABORAL EN MUJERES DUEÑAS DE PYMES EN LA CIUDAD DE MÉRIDA, YUCATÁN.

\section{Satisfacción relación estilo de vida-trabajo}

Con respecto a la relación estilo de vida-trabajo, se tuvieron ocho reactivos los cuales fueron:

1. Mi trabajo me ha permitido llevar el estilo de vida que aspiro.

2. He sentido que mi círculo social ha disminuido en los últimos meses.

3. El estrés del trabajo no me permite disfrutar momentos agradables con mis amigos/familia.

4. He preferido quedarme trabajando que salir con mis amigos/familia.

5. He podido llevar una actividad extra antes o después de mi trabajo (Gimnasio, yoga, artes, canto).

6. Mi trabajo me permite tener momentos de ocio con mi familia/amigos (cine, teatro, parrilladas).

7. Los problemas laborales interfieren con mi estilo de vida.

8. Estoy demasiada cansada después del trabajo para ver a mis amigos con la frecuencia que quisiera.

En la Figura 7, se puede observar en el rubro uno que, de 48 mujeres encuestadas, 12 (25\%) mencionaron estar totalmente de acuerdo con el hecho de que su trabajo les ha permitido llevar la vida que ellas aspiraban, 15 (31.25\%) mencionan estar de acuerdo, 18 (37.5\%) ni de acuerdo ni en desacuerdo y 3 (6.25\%) en desacuerdo. En el rubro dos, 19 (39.58\%) de las encuestadas manifiestan estar totalmente de acuerdo con el hecho de que su círculo social ha disminuido en los últimos meses, 18 (37.5\%) estar de acuerdo, 8 (16.6\%) ni de acuerdo ni en desacuerdo y 3 $(6,25 \%)$ mencionaron estar en desacuerdo. Para el rubro tres, 15 (31.25\%) alegan estar totalmente de acuerdo con que el estrés producido por su trabajo no les permite disfrutar de momentos agradables con su familia y/o amigos, 21 (43.75\%) mencionan estar de acuerdo y 12 $(25 \%)$ ni de acuerdo ni en desacuerdo. En el rubro cuatro, 22 (45.83\%) de las encuestadas afirman estar totalmente de acuerdo con el hecho de que preferir quedarse trabajando que salir con su familia y/o amigos, 16 (33.3\%) están de acuerdo, 10 (20.83\%) ni de acuerdo ni en desacuerdo, 6 mencionan estar en desacuerdo y solo 4 en total desacuerdo. Para el rubro cinco, 22 (45.83\%) de las mujeres aseguran estar totalmente de acuerdo con el hecho de que han podido llevar una actividad extra antes o después del trabajo (gimnasio, yoga, arte, canto, etc.), 16 (33.3\%) mencionan estar de acuerdo y 10 (20.83\%) ni de acuerdo ni en desacuerdo. En el rubro seis, 29 (60.41\%) mencionan estar totalmente de acuerdo con el hecho de que su 
ÉXITO Y SATISFACCIÓN LABORAL EN MUJERES DUEÑAS DE PYMES EN LA CIUDAD DE MÉRIDA, YUCATÁN.

trabajo les permite tener momentos de ocio con su familia y/o amigos (cine, teatro, parrilladas, etc.), $15(31.25 \%)$ de afirman estar de acuerdo y solo $4(8.33 \%)$ ni de acuerdo ni en desacuerdo. Para el rubro siete, 26 (54.16\%) alegan estar totalmente de acuerdo con el hecho de que sus problemas laborales han interferido con su estilo de vida, $20(41.66 \%)$ están de acuerdo y solo 2 (4.16\%) ni de acuerdo ni en desacuerdo. Finalmente, en el rubro ocho, 34 (70.83\%) del total de encuestadas asegura estar totalmente de acuerdo con el hecho de que después de su trabajo se sienten demasiado cansadas como para ver a sus amigos con la frecuencia que quisieran, 12 (25\%) mencionan estar de acuerdo con esto y solo $2(4.16 \%)$ ni de acuerdo ni en desacuerdo.

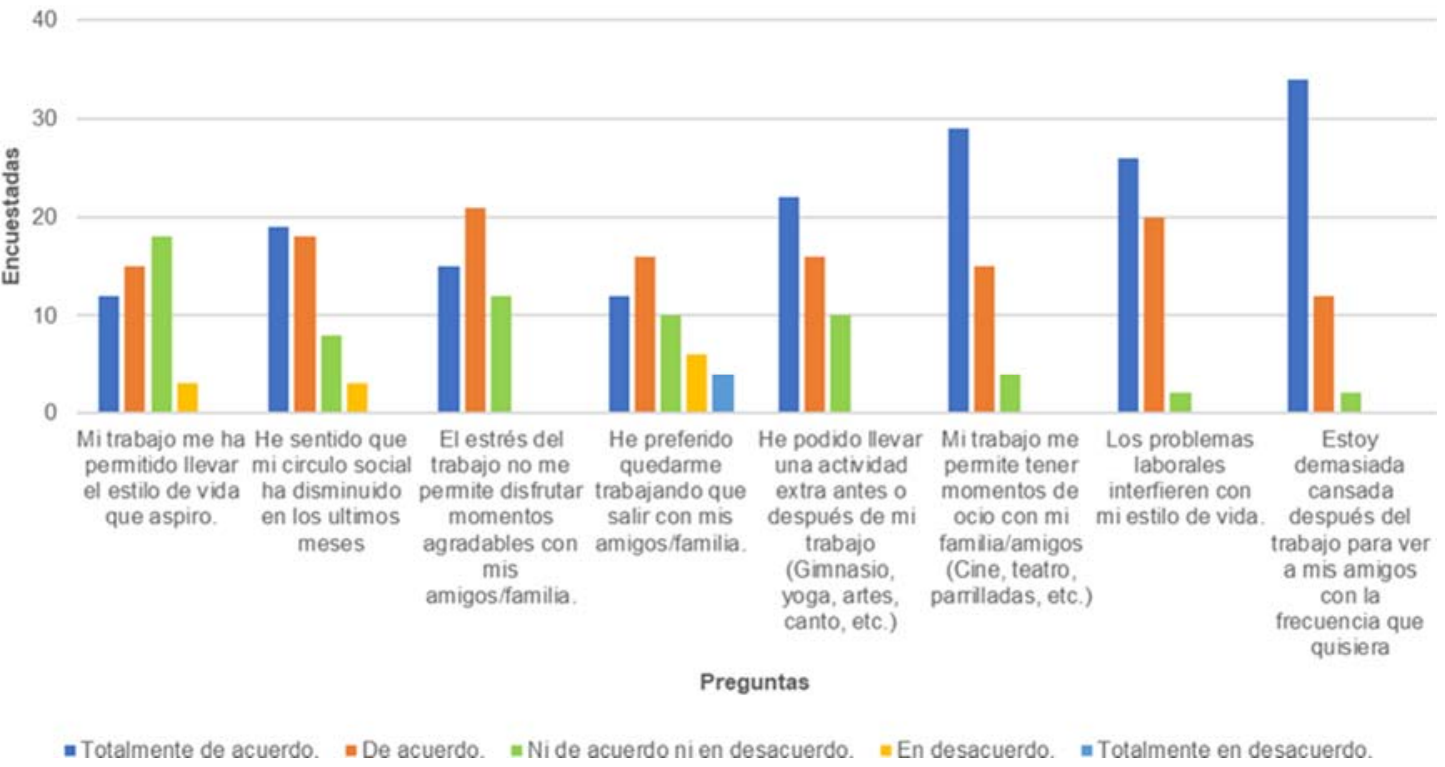

Figura 7. Relación estilo de vida-trabajo.

Fuente: Elaboración propia.

\section{DISCUSIÓN}

Estos resultados exponen que las mujeres que tienen un hijo y se encuentran en el rango de 50 años o más son las que poseen un nivel de satisfacción laboral más alto.

Se podría asumir, que las mujeres de 50 años o más, no tienen a su cargo niños pequeños y por ello tienen menos responsabilidades domésticas y pueden hacer un mejor balance de su desarrollo profesional y personal. 
ÉXITO Y SATISFACCIÓN LABORAL EN MUJERES DUEÑAS DE PYMES EN LA CIUDAD DE MÉRIDA, YUCATÁN.

Por otro lado, los resultados apuntan a que las mujeres tienden a tener una mejor satisfacción personal cuando se convierten en madres y el trabajo doméstico no afecta directamente a su trabajo.

Estos hallazgos, coinciden con lo encontrado por Carlier (2014) en Bogotá donde concluye que la percepción de satisfacción personal está correlacionada positivamente con la satisfacción laboral y con el éxito personal, no obstante, se ve afectada negativamente cuando las mujeres ceden su trabajo doméstico y cuidado de sus hijos a otras personas. Estos resultados muestran que las mujeres deben hacer trade-off entre los dos escenarios: su casa y su trabajo.

\section{CONCLUSIONES}

A través de los resultados obtenidos en esta investigación, y mediante el análisis de cada una de las variables caracterizadoras de las mujeres (que formaron parte de la investigación), se pudo determinar que el $48 \%$ de las mujeres dueñas de una pyme respecto a su relación empresa-persona, son mujeres con un rango de edad de 50 años o más, el $92 \%$ de estas mujeres contaba con al menos un hijo y su situación legal era casada, en relación al ambiente laboral de la empresa, siendo este el rubro más bajo (2.1240) se puede observar que el $74 \%$ de las mujeres que indicaron tener un buen ambiente laboral se encontraban en el rango de edad de 31 a 40 años, solamente el $43 \%$ tenía al menos un hijo y solamente el $24 \%$ eran casadas, con respecto al rubro 3, el cual corresponde a la satisfacción mediante la relación hogar-trabajo, se observa que el $87 \%$ de las mujeres menciona tener una alta satisfacción, siendo el rubro de mujeres con 50 años o más, de las cuales 95\% tenían al menos un hijo y $86 \%$ manifestaron tener una empresa de 20 a 50 empleados, de las cuales $47 \%$ de estas ya no operan en la empresa, solamente como parte accionista, el último rubro el cual menciona la satisfacción con respecto a la relación estilo de vida-trabajo, el $67 \%$ de las encuestadas mencionó tener entre 41 a 50 años, de estas el $67 \%$ contaba con al menos un hijo y el $34 \%$ de las empresas estaban en el rango de 20 a 50 empleados.

Este trabajo, contribuye a la discusión sobre los retos que las mujeres empresarias afrontan en el ámbito laboral y hace una aporta evidencia empírica sobre el tema, lo cual es relevante dada la escasez de estudios de este tipo tanto en Yucatán como en México.

Es importante mencionar, que la pandemia generada por el virus SARS-CoV-2 propició una reducción en las muestras establecidas, limitando así el estudio y el tamaño de la población se 
ÉXITO Y SATISFACCIÓN LABORAL EN MUJERES DUEÑAS DE PYMES EN LA CIUDAD DE MÉRIDA, YUCATÁN.

tomaron algunas decisiones sobre la información que se recogió en aras de construir los índices de percepción.

Se considera pertinente resaltar la importancia de hacer más estudios sobre el tema para poder profundizar en las relaciones entre los diferentes índices de satisfacción y éxito, así como de los elementos que inciden en ella.

\section{REFERENCIAS BIBLIOGRÁFICAS}

Barrios, A. y Barrios, O. (2016). Participación femenina en el mercado laboral de México. México. Universidad Autónoma del Estado de México. Recuperado de http://economia.uaemex.mx/Publicaciones/e903/Yeni_y_Oscar.pdf

Castillo, D. (2001). Los nuevos precarios, ¿mujeres u hombres?: Tendencias en el mercado de trabajo urbano en Panamá, 1982-1999. Papeles de población, 7(27), 99-145. Recuperado de

http://www.scielo.org.mx/scielo.php?script=sci_arttext\&pid=S140574252001000100006\&ln $g=e s \& t \operatorname{lng}=e s$.

CEPAL. (2010). Igualdad de género: participación, autonomía y empoderamiento de las mujeres del nuevo informe de las Naciones Unidas sobre la región: América Latina y el Caribe avanza hacia los Objetivos del Milenio. Capítulo V. Recuperado de http://www.cepal.org/oig/noticias/noticias/9/40069/2010_622_ODM_ESPANOL_CapV.pdf

Duarte, J. M. y García, J. B. (2016). Igualdad, Equidad de Género y Feminismo, una mirada histórica a la conquista de los derechos de las mujeres. Revista CS, (18),107-158. ISSN: 2011-0324. Disponible en: https://www.redalyc.org/articulo.oa?id=4763/476350095006

González, S. (2004). Igualdad de oportunidades entre mujeres y hombres en el mercado laboral. Universidad de Las Palmas de Gran Canaria. Recuperado de https://www2.ulpgc.es/hege/almacen/download/22/22044/ponenciasmgb.pdf https://www.inegi.org.mx

Neil, D. A. y Cortes, L. (2018). Procesos y fundamentos de investigación científica (Primera ed.). UTMACH. 


\section{Original}

ÉXITO Y SATISFACCIÓN LABORAL EN MUJERES DUEÑAS DE PYMES EN LA CIUDAD DE MÉRIDA, YUCATÁN.

Ramírez, V. H., Domínguez, D. P. y Vallejo, M. A. A. (2011). MSMEs craft as a means of development for rural groups in Mexico. 29.

Ruiz, L. J. C. (s. f.). Autoridades Universitarias. 110.

Texis, M., Ramírez, M., Aguilar, J. G. (2016). Microempresas de base social y sus posibilidades de supervivencia. Contaduría y administración, 61(3), 551-567. https://doi.org/10.1016/j.cya.2015.04.001

Todaro, R., Godoy, L. y Abramo, L. (2002). Desempeño laboral de hombres y mujeres: Opinan los empresarios. Cadernos Pagu, 17-18, 197-236. https://doi.org/10.1590/S010483332002000100008 